\title{
Following an RF trail to its source
}

\author{
Aseem Wadhwa ${ }^{\# 1}$, Upamanyu Madhow ${ }^{* 2}$, João Hespanha ${ }^{\# 3}$ and Brian M. Sadler ${ }^{* 4}$ \\ \# Department of ECE, University of California Santa Barbara, CA 93106 \\ 1 aseemeece. ucsb.edu \\ ${ }^{2}$ madhowlece.ucsb.edu \\ ${ }^{3}$ hespanhadece.ucsb.edu \\ * Army Research Laboratory, Adelphi, MD 20783 \\ ${ }^{4}$ brian.m.sadler6. civ@mail.mil
}

\begin{abstract}
We consider the problem of drawing a mobile platform such as an Unmanned Aerial Vehicle (UAV) to a radio frequency (RF) source surrounded by local scatterers. The UAV starts from a significant distance (e.g., kilometers) from the source, and uses received signal strength (RSS) measurements along its path to continue to adapt its trajectory. The fundamental bottleneck in using the RSS for this purpose is that large spatial variations in the the RSS due to multipath fading can swamp out the relatively smaller variations due to the change in the distance to the source. We characterize these variations using ray tracing, explicitly modeling the scattering environment around the source. We discover that the rate of spatial variations decreases as the direction of travel becomes more aligned with the line-of-sight (LoS) to the source. Thus, it is possible to get trapped in a very long fade which makes it difficult to gather information regarding distance to the source. We use these insights to devise a multiphase algorithm which first uses averages of RSS in multiple directions to estimate the "right" direction of travel towards the source, and then uses longer term averages to decide how long to proceed along the chosen direction. The distance traveled by the UAV to get to the source is, on average, about three times longer than the shortest path. While the proposed algorithm is inspired by the motion of bacteria in search of food, it has been significantly optimized to take into account the sensitivity of RSS to direction of travel.
\end{abstract}

\section{INTRODUCTION}

In this paper, we consider the problem of following an RF beacon to its source using a mobile platform. The RF beacon might be emitted by a sensor which would like to form a short-range, high data rate link to upload its measurements, or may be used, either in military or civilian settings, to draw in support for emergencies or for search and rescue. The specific scenario we consider is that of an unmanned aerial vehicle (UAV) starting a significant distance away (of the order of kilometers) from an RF source surrounded by local scatterers. The UAV uses RSS measurements along its path to adapt its trajectory so as to approach the source as quickly as possible. While a natural approach for this purpose is to follow the gradient of the RSS, the fundamental bottleneck is that large spatial variations in the RSS due to multipath fading can swamp out the relatively smaller variations due to the change in the distance to the source. Our objective is to see whether

Research supported by the Institute for Collaborative Biotechnologies through grant W911NF-09-0001 from the U.S. Army Research Office. The content of the information does not necessarily reflect the position or the policy of the Government, and no official endorsement should be inferred. we can follow the RF trail even in this challenging setting (angle of arrival information using antenna arrays at the UAV, for example, could significantly improve performance).The key contributions of this paper are to characterize the spatial variations of the RSS as a function of the trajectory, and to show this characterization to devise effective averaging mechanisms for estimating and updating the direction of travel towards the source.

Our model for RSS variations is based on ray tracing using the following propagation model. The RF beacon is on flat terrain surrounded by a few scatterers, but there are no scatterers around the UAV. The spatial model of fading that we obtain is found to be relatively insensitive to the number of scatterers. The spatial profile depends on the distance of the UAV from the beacon relative to the carrier wavelength: in our numerical results, we typically consider initial distances of the order of $1.5 \mathrm{~km}$ between the UAV and source, with the beacon being a tone at $2.4 \mathrm{GHz}$ (i.e., a wavelength of 0.125 $\mathrm{m})$, so that the initial distance is about 12000 wavelengths.

Our main results are summarized as follows. We find that the rate of spatial variations of the RSS along a straight line path depends on the approach angle, defined here as the angle between the current path and the LoS to the beacon. In particular, the spatial variations along different angles are self-similar, getting spatially dilated as the approach angle decreases. Thus, as the direction of travel becomes more aligned with the LoS, the rate of spatial variations decreases drastically, and it is possible to get trapped in a very long fade which makes it difficult to gather information regarding distance to the source. We use these insights to devise a multiphase algorithm which first uses averages of RSS in multiple directions to estimate the "right" direction of travel towards the source, and then uses longer term averages to decide how long to proceed along the chosen direction. The distance traveled by the UAV to get to the source is, on average, about three times longer than the shortest path. Our algorithm is inspired by the motion of bacteria in search of food (termed chemotaxis), which also interleaves straight line runs with abrupt changes in direction. While it is shown in [1] that this type of algorithm (which we term optimotaxis) is very robust to local extrema and noisy measurements, general-purpose optimotaxis can result in slow convergence. The RSS-adapted optimotaxis algorithm proposed in this paper accounts for the 
structure of the RSS landscape to speed up convergence, and results in substantial performance improvements over generalpurpose optimotaxis, taking about eight times less time on average to successfully reach the source.

The rest of the paper is organized as follows. In Section II we describe the ray tracing model in detail. In Section III we look at the how the RSS varies over space. In Section IV the algorithm proposed for routing the UAV is discussed. Section $\mathrm{V}$ gives the simulation results of the algorithm and the paper is concluded with a mention of future work in Section VI.

\section{Related work}

Most of the literature on RSS-based tracking focuses on localization and tracking of (possibly moving) objects using stationary sensors [2][3][4][5][6]. In contrast, we use a moving RSS sensor to track a stationary source (evaluating and adapting the proposed algorithm for moving sources is an important topic for future work). Examples of prior work on modeling spatial variations in RSS include [7][8], which discuss methods to build RSS maps around a source using measurements by a group of robotic agents, and [9] [10], which use outdoor experiments to study the impact of factors such as distance, antenna orientation, and antenna height on RSS. However, the literature does not provide insight into the spatial variations of RSS at long ranges and as a function of angle, as required by the tracking problem considered here.

Recent work that does consider, as we do, RSS-based tracking using a mobile sensor appears in [11][12]. The major difference from our work is that it considers robotic platforms using gradient-based tracking over a few meters, in contrast to the ranges of hundreds of meters considered in our work, where the lengths of the fades make gradient-based tracking infeasible. A maximum likelihood estimate for the RSS gradient [11][12] is found to be effective as long as the SNR is high enough and when the sensor is close enough to the source. Other prior work on RSS gradient estimation includes [13][14].

\section{Propagation MOdel}

The source is located at the origin of a 2-D plane. We are interested in finding the value of RSS at a point $\mathrm{P}$ in space with polar coordinates $(r, \alpha)$. There are $T$ point reflectors situated along a ring of radius $R$ centered at the origin. The angular positions of the reflectors $\phi=\left[\phi_{1}, \ldots \ldots ., \phi_{T}\right]$, is randomly chosen but then kept fixed. The model is similar to Clarke's model [15]. The signal received at point $\mathrm{P}$ consists of a line of sight (LoS) component corresponding to the direct path between the transmitter and receiver, and $T$ non-LoS (NLoS) components corresponding to reflections from scatterers. The electric field at point $\mathrm{P}$ is given as

$$
\begin{gathered}
E F(r, \alpha)=\frac{e^{-j \beta r}}{r}+\sum_{i=1}^{T} \frac{\Gamma(i) e^{-j \beta l_{i}}}{l_{i}} \\
l_{i}=\sqrt{R^{2}+r^{2}-2 \operatorname{Rrcos}\left(\phi_{i}-\alpha\right)}+R
\end{gathered}
$$

where $\beta=\frac{2 \pi}{\lambda}, \Gamma(i)$ is the complex reflection coefficient for reflector $i$ [16] and $l_{i}$ is the path length of the ray coming from the $i^{\text {th }}$ reflector, obtained from cosine law (Fig. 1). The RSS in $\mathrm{dB}$ is given by

$$
R S S(r, \alpha)=20 \log _{10}|E F(r, \alpha)|
$$

Since we assume the source to be stationary, the value of RSS at a point does not change with time (but the UAV sees time-varying RSS due to its motion).

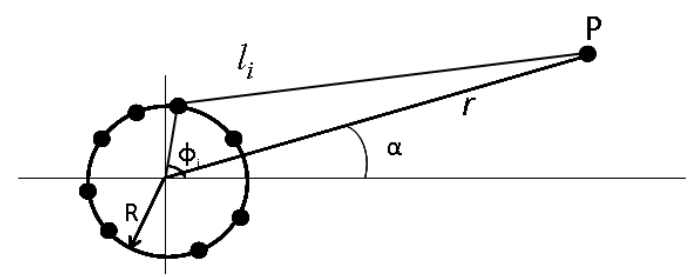

Fig. 1: Reflectors on a ring around the source

\section{SPATIAL RSS PROFILES: OBSERVATIONS}

In this section, we investigate the spatial variations of RSS through ray tracing simulations, based on the model described in the previous section. We also attempt to provide analytical insight based on the expression in (1). The values of $(T, R, \phi)$, which determinesthe reflector locations, are kept fixed. The $\mathrm{RF}$ beacon is at frequency $2.4 \mathrm{GHz}$, so that the wavelength is $\lambda=1 / 8 m=0.125 \mathrm{~m}$. We set $T=20, R=80 \lambda=10 \mathrm{~m}$, and choose $\phi$ to be uniformly random over $[0,2 \pi]$. Later in the section we look at the effect of changing the values of these parameters. We use the complex permittivity of concrete wall [16] for calculating the reflection coefficients $\Gamma(i)$ as a function of the angle of incidence.

Fig. 3a shows a 3-D plot of RSS variations over a plane of size $2000 \mathrm{~m} \times 2000 \mathrm{~m}$. The regime of interest is $r \gg R$, where $r$ is the distance of the UAV from the source (Fig. 2 ). Thus, we are interested in the spatial profiles of RSS over $r \sim 200 m-2000 m$. We are interested in determining how the RSS changes as the UAV moves along various directions. Fig. $3 \mathrm{~b}$ shows the RSS profile in a much smaller region - a square of size $100 \mathrm{~m} \times 100 \mathrm{~m}$ around $r=900 \mathrm{~m}$. We observe that the profile consists of hills and valleys radiating from the center (source). As the UAV travels in directions perpendicular to the radial lines, it encounters hills and valleys at a much higher rate than when it travels inwards/outwards towards the center. In Fig. 4 we examine the RSS measurements along straight lines at different angles to the direction pointing towards the source; we term the angle between such a straight line and the LoS to the source the approach angle. Two key observations are worth making, based on these plots. First, the relative change in the path lengths for the multiple reflected paths as a function of distance travelled is smaller for small approach angles, resulting in very slow fading. Thus, we can get deep and long fades that last over hundreds of wavelengths of travel. This is very different from the far more rapid fading 
patterns that would be seen in rich scattering environments, which is often the situation of interest in many communication applications. Second, variations due to fading are significantly larger than the small changes in RSS due to changes in the distance to the source. The power scales as $1 / r^{2}$ in our model, so that the relative change in $\mathrm{RSS}$ scales as $1 / r$ : when traveling a distance $x$ towards the source, the fractional change in RSS is given by $\frac{\Delta(R S S)}{R S S}=\frac{-2 x}{r}$ if there is no fading. Thus, at the large ranges of interest, the useful information in the RSS (regarding changes in $r$ ) is small, and easily swamped by variations due to fading. Table I shows, for two typical ranges of interest, the change in RSS values expected in the absence of fading (i.e., with only the LOS component) as we travel a distance $x$ ranging from $40-100 \mathrm{~m}$. These path loss based increments (which carry the useful information regarding changes in $r$ ) are clearly very small, and hence easily swamped by variations due to fading.

These two observations illustrate why our problem of following the RF trail is so challenging. One would like to move in the direction towards the source to get the largest path loss based increments in RSS, but these are the very directions most severely affected by fading. Directions perpendicular to the 'correct,' or LoS, direction give much faster fades (of the order of $40 \lambda-80 \lambda$ at $r=800 \mathrm{~m}$ ) which can be averaged out more easily, but provide little change in the path loss based component of RSS.

Figures 5 and 6 show views of the RSS landscape. Fig 5 shows the RSS variation as UAV moves around a circle of radius $r=800 m$ centered at the origin, with $\alpha$ varying from 0 to $2 \pi$. Angular size of the fades (distance between 2 peaks) vary roughly between $.006-.012$ radians, which corresponds to a variation of $40-80 \lambda$ at this radius $(r=800 m)$. Let us call these quantities $\theta_{\text {peak }}$, angular separation of 2 peaks and $d_{\text {peak }}$, separation between 2 peaks in metres. As has been observed through simulations, for a fixed value of $T$, the number of scatterers and $R$, the radius of the ring, the value of $\theta_{\text {peak }}$ remains in approximately the same range. For $T=20, R=$ $10 \mathrm{~m}, \theta_{\text {peak }} \sim .006-.012$ ) independent of $r$, as long as $r$ is sufficiently larger than $R$. Since $d_{\text {peak }}=r \cdot \theta_{\text {peak }}$, the distance between peaks decreases as we approach the source, and it becomes easier to spatially average out fades. Fig. 6 shows RSS variations as $r$ varies from $1500 m$ to $200 m$, keeping $\alpha$ fixed.

\begin{tabular}{|c|c|c|c|c|}
\hline$x$ & $40 m$ & $60 m$ & $80 m$ & $100 m$ \\
\hline \hline$r=1000 m$ & $0.35 d B$ & $0.54 d B$ & $0.72 d B$ & $0.92 d B$ \\
\hline$r=500 m$ & $0.72 d B$ & $1.11 d B$ & $1.51 d B$ & $1.94 d B$ \\
\hline
\end{tabular}

TABLE I: Changes in path loss component of RSS over distances 40-100m

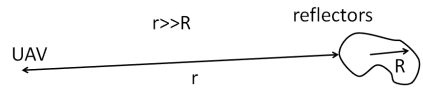

Fig. 2: Regime of interest

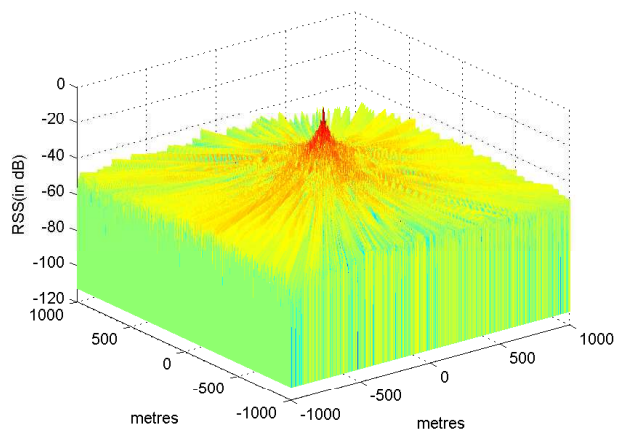

(a)

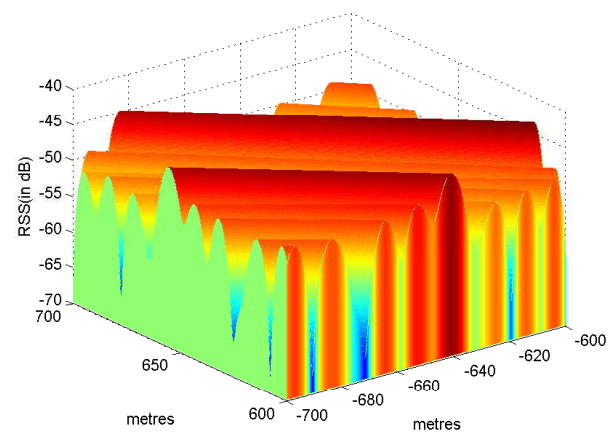

(b)

Fig. 3: Plots of RSS variation over space
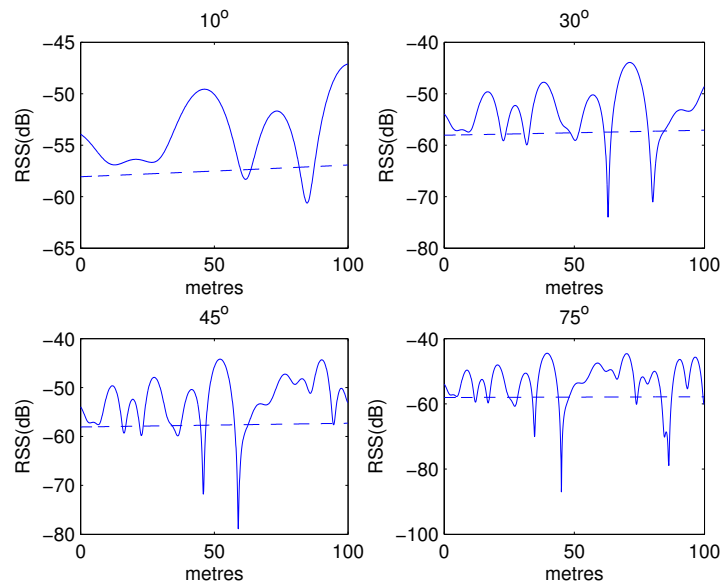

Fig. 4: Typical RSS profiles while moving towards the source from $800 \mathrm{~m}$ away at different angles of approach, for a distance of $100 \mathrm{~m}$ ( 800 wavelengths) [dotted lines show only the LOS component] 


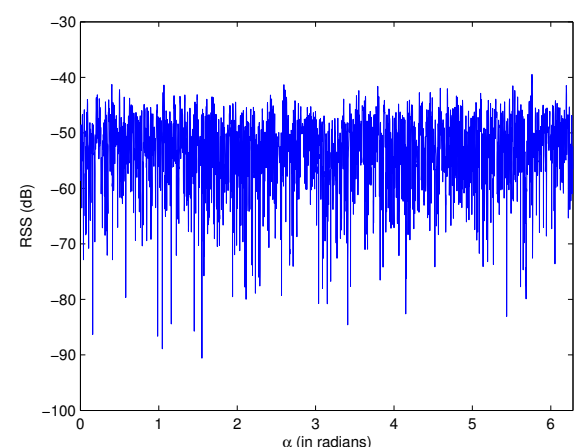

Fig. 5: Variation of RSS around a circle of radius $800 \mathrm{~m}$
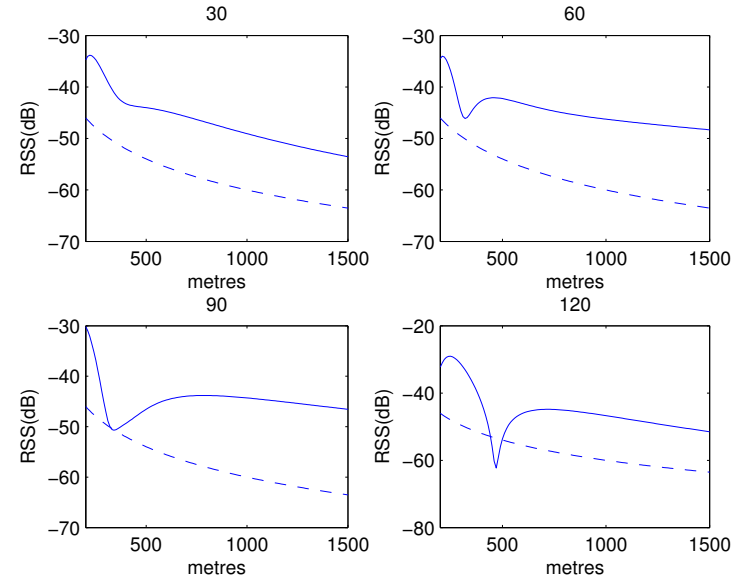

Fig. 6: Variation of RSS with $r$, for different fixed values of $\alpha$ (dotted line - just the LOS component)

\section{Analytical Insight}

We begin by deriving an approximation for the electric field in (1) for $r \gg R$.

$$
\begin{gathered}
E F(r, \alpha) \approx \frac{e^{-j \beta r}}{r}+\sum_{i=1}^{T} \frac{\Gamma(i) e^{-j \beta\left(r-R \cos \left(\phi_{i}-\alpha\right)+R\right)}}{r} \\
E F(r, \alpha) \approx \frac{e^{-j \beta r}}{r}\left[1+\sum_{i=1}^{T} \Gamma(i) e^{-j \beta R\left(1-\cos \left(\phi_{i}-\alpha\right)\right)}\right] \\
10 \log \left(|E F(r, \alpha)|^{2}\right) \approx 10 \log \left(\frac{1}{r^{2}}\right)+ \\
10 \log \left(\left|1+\sum_{i=1}^{T} \Gamma(i) e^{-j \beta R\left(1-\cos \left(\phi_{i}-\alpha\right)\right)}\right|^{2}\right)
\end{gathered}
$$

The first term in (6) corresponds to path loss, and the second to fading. The path loss term does not depend on $\alpha$, while the fading term is independent of $r$ (ignoring the dependence of $\Gamma$ on $r$ and $\alpha$ ). Now, suppose that the UAV moves from point A to point $\mathrm{B}$ as shown in Fig. 7, for a distance $x$ along a straight line at approach angle $\theta$ (we are ignoring the small change in $\theta$ as we move along the path), then it sweeps an angle of $\triangle \alpha(x)$ given by (assuming $r \gg x$ )

$$
\begin{gathered}
\alpha(x)=\alpha_{\text {initial }}+\tan ^{-}\left(\frac{x \sin \theta}{r-x \cos \theta}\right) \approx \alpha_{\text {initial }}+\frac{x \sin \theta}{r} \\
\triangle \alpha(x) \approx \frac{x \sin \theta}{r} \\
r(x)=\sqrt{r^{2}+x^{2}-2 r x \cos \theta} \approx r
\end{gathered}
$$

Thus $\triangle \alpha(x)$ varies as $\frac{x \sin \theta}{r}$. This indicates that, for small angles $\theta$, the change in angle subtended at origin is slow as a function of $x$, while the change is maximized for $\theta=90^{\circ}$. Under this approximation, the RSS variations along different approach angles are self-similar, obeying the following scaling:

$$
r \operatorname{ss}_{\theta}(x)=r \operatorname{ss}_{90^{\circ}}(\sin \theta \cdot x), \quad r \gg x
$$

where $r s s_{\theta}(x)$ is the RSS along approach angle $\theta$. The preceding scaling corresponds to spatial dilation of the RSS profile as a function of $x$ by the factor $1 / \sin \theta$, as evident from the plots in Fig. 4. In addition, we also see a scalar translation of the RSS profile; this is due to the dependence of $\Gamma$ terms on $r$ and $\alpha$. Fig. 8 shows the variation of distances between 2 consecutive fading peaks as a function of $\theta, d_{\text {peak }} / r \propto 1 / \sin \theta$.

The preceding results indicate that it is crucial to take into account the spatial frequency of the fading in order to estimate and control the UAV's trajectory. In the next section, we propose an algorithm that builds on these insights.

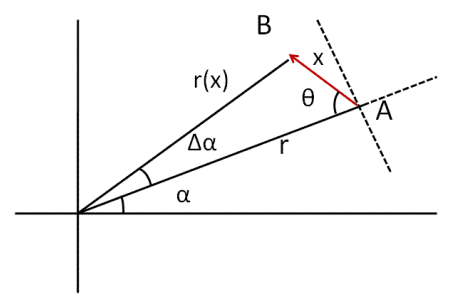

Fig. 7: Moving along a direction at an angle $\theta$ to the 'correct' direction

\section{Effects of changing $T, R, \lambda$}

Increasing $T$, the number of scatterers around the source has a negligible effect on the broad observations we have made regarding RSS variation. When $T$ is small i.e. less than 34 , then the variance of $\theta_{\text {peak }}$, angular size of the fades, is large because the fades are sensitive to angular location of the UAV relative to the reflectors and the source. However, these effects rapidly dissipate as $T$ increases, and the nature of RSS variations is essentially unchanged for $T>5$.

The RSS profile scales with the carrier wavelength $\lambda$, and depends on the normalized parameters $x / \lambda, r / \lambda, R / \lambda$. 


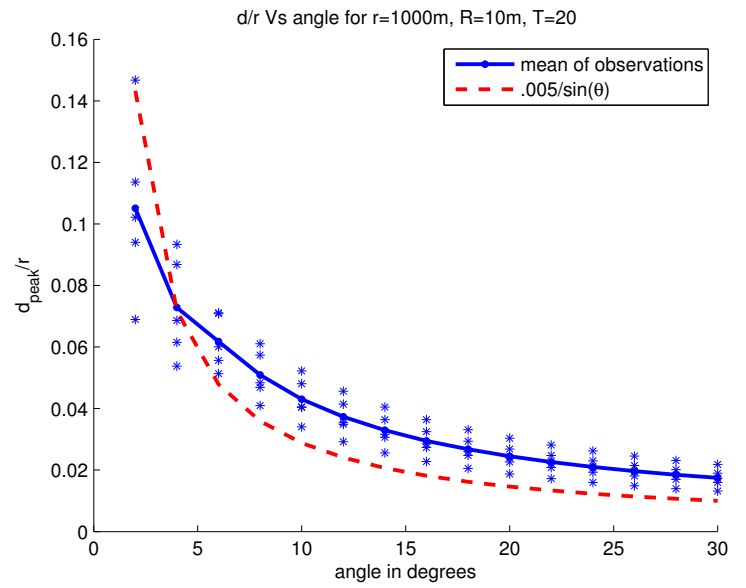

Fig. 8: Variation of $d_{\text {peak }} / r$ with $\theta$, the angle of approach

Furthermore, the lengths of fades (normalized by $\lambda$ ) is a function of $r / R$; for example, the RSS variations are similar for $R=80 \lambda(10 m), r=6400 \lambda(800 m)$, and for $R=$ $40 \lambda(5 \mathrm{~m}), r=3200 \lambda(400 \mathrm{~m})$. In particular, the fade length increases with $r / R$ : the angular distance between fading peaks varies inversely as $R$ when we keep $r$ fixed: $\theta_{\text {peak }} \sim 1 / R$, so that the distance between peaks $d_{\text {peak }}=\theta_{\text {peak }} \cdot r \sim r / R$. For example, fixing $r=800 \mathrm{~m}$, we obtain $d_{\text {peak }} \sim 30-70 \lambda(4 \mathrm{~m}-$ $9 m)$ for $R=10 m$, and $d_{\text {peak }} \sim 60-140 \lambda(8 m-18 m)$ for $R=5 m$.

\section{Observations robust - ring not centred at source, walls}

The basic observation that fades are longer in the LoS direction (for $r \gg R$ ) is quite robust to minor variations in our scattering model, such as shifting the centre of the ring of scatterers away from the source or replacing the ring reflectors by a several finite length line reflectors (representing walls) which are not symmetrically placed with respect to the source.

\section{Algorithm FOR UAV ROUTING}

The objective is to devise a strategy for routing the UAV, which starts at a distance $r_{\text {initial }}$ (taken to be $1500 \mathrm{~m}$ in our simulations) away from the source, to get within $150 \mathrm{~m}$ $\left(d_{\text {terminate }}\right)$ of the source as quickly as possible. We assume constant velocity throughout the UAV flight, ignoring speed variations during turns. Thus, time of flight is directly related to distance travelled and we strive to minimize this.

The problem of tracking a source by an autonomous vehicle, which has only RSS measurements to rely on, is very similar to the source seeking problem discussed by Mesquita et al in [1]. In this work, a bio-inspired optimization approach called optimotaxis is used to control a group of autonomous agents that seek to locate the maximum of an underlying sensed signal. Optimotaxis works without knowledge of position or gradient measurements and has been proven to work even in the case of signal profiles with multiple maximas. Hence it is natural to apply optimotaxis to our present problem, and we first report on some typical results from doing this. We then show that it is possible to significantly improve its performance by exploiting the insights we have gained regarding the spatial variations of RSS.

\section{A. Optimotaxis}

In optimotaxis, one or more autonomous vehicles/agents perform a biased random walk, analogous to the chemotaxis procedure used by bacteria seeking food. (For the problem at hand, there is only one agent.) The agents switch between 2 alternate behaviors called run and tumble. Each agent travels with a constant velocity in the run state for some time and then tumbles i.e. shifts to another run state in a different direction. The duration of runs is a function of a sensed scalar signal: the probability of a tumble depends on the improvement seen in the signal values. The control law to decide at time $t$ whether to tumble or not, is a function of signal measurements up to time $t$. For searching in two dimensions, the signal measured at time $t$ is denoted by $F(x(t), y(t))$, where $(x(t), y(t))$ is the location of the agent at time $t$. The goal is to devise a control law so that the probability distribution of the agent converges to a pre-specified "shaping" function of $F$. For example, by choosing this shaping function as $Q(x, y)=F^{n}(x, y)$, where $n$ is large, the density converges close to the maximum of $F$. Convergence is assured under certain conditions of boundedness for the underlying function $F(x, y)$ [1]. For a single agent, the average of its positions over time matches the signal profile. We choose the shaping function such that it is possible to normalize it to obtain a valid probability density.

Optimotaxis for RF beacon tracking: We take the underlying function $F(x, y)$ to be a spatial average of the RSS, where averaging is done along path being followed by the UAV in the run state. We use a shaping function to raise $F$ by a power of $n(n \sim 6-8)$ to accentuate the maxima which is at the origin/source. The criterion to tumble at any time $t$ is then given by [1]

$$
\frac{q(x(t), y(t))}{q\left(x\left(t_{k}\right), y\left(t_{k}\right)\right)} e^{\frac{-\left(t-t_{k}\right)}{\eta}} \leq u, \quad t \geq t_{k}
$$

That is, the UAV tumbles or changes its direction to a new random direction at time $t$, if the preceding condition is satisfied; where $q(x(t), y(t))=\left(\int_{t-T}^{t} r s s(x(t), y(t))\right)^{n}$, $T$ denotes the averaging interval; $u$ is a random variable uniformly distributed over $[0,1]$, and $\eta$ is a constant chosen using offline optimization of algorithm performance.

Results of Optimotaxis: While convergence was achieved in almost all the cases simulated, most of the times the distance UAV has to travel before it reaches near the source is very large. A sample tracking path is shown in Fig. 9. In this particular example, the UAV had to travel about 10 times the shortest distance from its initial position to the source. In fact, this ratio is typically significantly larger than 10 in most of the simulations that we have run. The problem is that the UAV is not able to continue in the right direction for too long: the presence of long and deep fades in the signal profile can make a wrong direction appear 'good' for 100s of meters, and vice versa. While the proportion of time the UAV spends in a 
region depends on the relative magnitude of the signal there and eventually it does reach close to the beacon (at which point it is assumed to stay there), as we show next, it is possible to obtain significant improvements by exploiting the spatial rates of RSS variations in different directions.

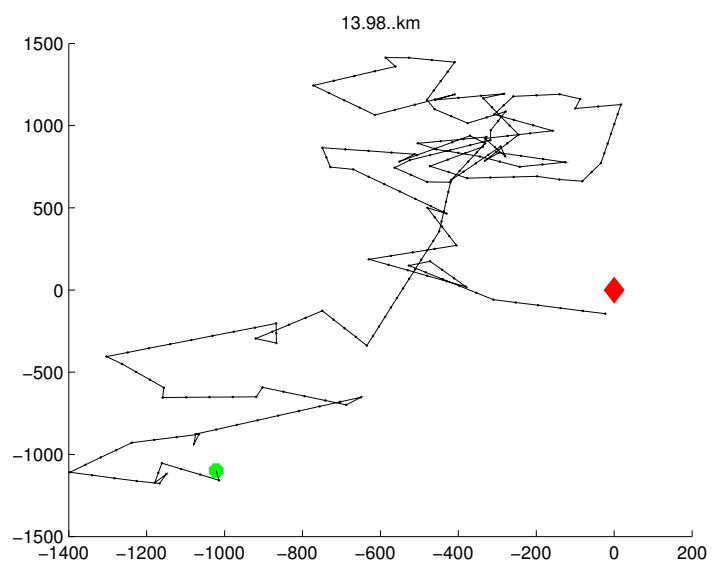

Fig. 9: An instance of general-purpose optimotaxis applied to tracking an RF source at the origin, starting from a distance of about $1.5 \mathrm{~km}$. The path length in this instance is $14 \mathrm{~km}$ (green circle is the starting point and source is at the red dot (origin))

\section{B. RSS-Adapted Optimotaxis}

We now adapt optimotaxis based on what we now know about the RSS landscape. The tracking strategy in our RSSadapted optimotaxis algorithm is divided into 3 stages. The first two stages try to obtain an estimate of the direction of the source. In the third stage, the UAV moves in the estimated direction while things are "going well." Once the improvement is found to be not good enough, the UAV executes the first two steps again to find a new direction. The algorithm is similar in spirit to the run and tumble in general-purpose optimotaxis, except that instead of choosing a new direction at random, we expend some time estimating the new direction to go in. Step 1 uses spatial frequency information to estimate the orientation of the source i.e. it produces an estimate of a line along which the source may lie. However, it does not tell us which direction along this line the source is, and we employ Step 2 to estimate the correct "half" of the line. We do not expect to make any net progress towards the source in these two steps, hence the objective is to travel as little as possible to achieve estimates of acceptable quality. Step 3 is when the UAV makes progress towards the target. However, since there is error attached to the estimated direction, which is only going to increase as the UAV travels, it continues only while sufficient improvements in RSS are seen. As we approach the source, new direction estimates are required, and the UAV goes into the "estimation" mode of Steps 1 and 2. The three steps are explained in detail in the following subsections.

\section{Step 1: Estimating the orientation of source}

The UAV "walks" along a few different directions, each for a fixed distance $d_{\text {walk }}$, observing and recording the RSS. In our implementation, we sample 4 directions as shown in Figure 10: the first is randomly chosen, the second is perpendicular to the first, and the two other directions are chosen mutually perpendicular and rotated $45^{\circ}$ with respect to the first two. The Fourier transforms of the 4 recorded RSS signals are compared and the one with the lowest frequency content is estimated to be the one most closely aligned with the LoS towards the source. The value of $d_{\text {walk }}$ trades off estimation accuracy versus overhead, and simulations show that $d_{\text {walk }}=20 \mathrm{~m}$ (corresponding to an overhead of $d_{\text {step } 1}=4 \cdot d_{\text {walk }}=80 \mathrm{~m}$ each time Step 1 is carried out) works well in our setting. An important topic for further investigation is to develop analytical guidelines for choice of $d_{\text {walk }}$.

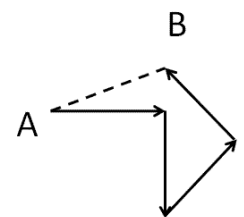

Fig. 10: Step 1: 4 direction walk

\section{Step 2: Estimating the correct direction}

Step 1 provides a (relatively coarse) estimate of the line along which the source lies, up to the resolution of our angular sampling scheme: sampling in 4 directions gives the worst case error of $22.5^{\circ}$ and an average error of about $12^{\circ}$. However, the spatial frequency of fades used in this step does not tell us which half of the line to proceed on in order to approach the source. In Step 2, we resolve this ambiguity by exploring one half at random before committing to a longer "run" along one side of the line in Step 3.

Let $p\left(d_{\text {step } 2}\right)$ denote the probability of being correct in Step 2. Since the initial selection of which side to explore is random, the expected value of progress made towards the source is going to be 0 irrespective of the value of $d_{\text {step } 2}$ or the choice of exploration trajectory. Thus, the objective of Step 2 is to find a path that minimizes $d_{s t e p 2}$, subject to the constraint $p\left(d_{\text {step } 2}\right) \geq 0.9$ (say) . Constraining ourselves to piecewise linear trajectories for simplicity, we have considered candidates such as those shown in Figure 11. Due to lack of space, we omit detailed discussion of these schemes, but state only that method (c) offers the best performance among the ones considered. This method averages out the fading along the direction of the highest rate of change (perpendicular to the estimated LoS) by going a distance $d_{V}$ "vertically" along $\mathrm{AB}$. Averaging along $\mathrm{AB}$ gives an estimate of RSS at distance $r$, while averaging along $\mathrm{CD}$ gives an estimate of RSS at distance $r \pm d_{H}$ from source. The distance $d_{V}$ must be large enough to provide sufficient averaging over fades, while $d_{H}$ must be large enough so that the change in RSS due to 
range difference is not swamped by the residual fading. We find through simulations that $d_{V}=150 \mathrm{~m}$ and $d_{H}=25 \mathrm{~m}$ (corresponding to $d_{\text {step } 2}=2 \cdot d_{V}+d_{H}=325 \mathrm{~m}$ ) give $p\left(d_{\text {step } 2}\right)$ better than 0.8 in most circumstances.

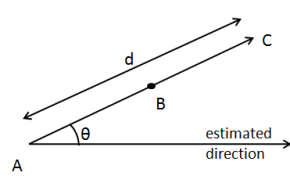

(a)

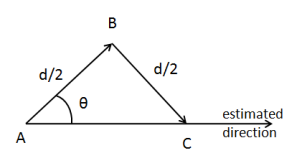

(b)

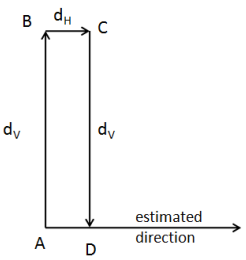

(c)

Fig. 11: Some candidate trajectories for Step 2

\section{Step 3: Making Progress}

This is the step in which the UAV makes a 'run' in the direction determined by the previous 2 steps. As it flies along this direction, it computes the average RSS with a sliding window of size $s_{w}$, arss $(x)=\int_{x-s_{w}}^{x} r s s(x) d x$. That is, $\operatorname{arss}(x)$ is a low pass filtered version of the RSS. The UAV "sprints" for a minimum distance of $d_{\text {sprint }}$. After this it keeps flying in this direction only while improvements in the averaged RSS are seen, and initiates a change of direction (after rerunning Steps 1 and 2) if the average filtered RSS decreases over an observation interval $I_{o}$. The value of $d_{\text {sprint }}$ is at least $s_{w}+I_{o}$. The algorithm terminates as soon as the $\mathrm{UAV}$ is within distance $d_{\text {terminate }}$ distance of the source.

\section{Simulation Results}

The simulation parameters are given in Table II. Figure 13 shows a few instances of the tracking path followed by the UAV using RSS-adapted optimotaxis. Fig. 12 shows the histograms of path lengths for optimotaxis and RSS-adapted optimotaxis. The average tracking path length for RSS-adapted optimotaxis is found to be $4.3 \mathrm{~km}$, which is about 3 times of the shortest approach path $(1.5 \mathrm{~km})$ and about 8 times shorter than that of general-purpose optimotaxis.

\section{CONCLUSIONS}

Our ray tracing based propagation model provides fundamental insights on the spatial variations of RSS, and reveals their strong dependence on the approach angle for the scenario considered here, where local scatterers around the source produce strong fades at a distant location away from the clutter. The multimodal nature of the RSS field precludes simple gradient based algorithms for following the RF trail. The bioinspired optimotaxis algorithm does succeed in handling the local extrema of RSS, but is quite inefficient, requiring the

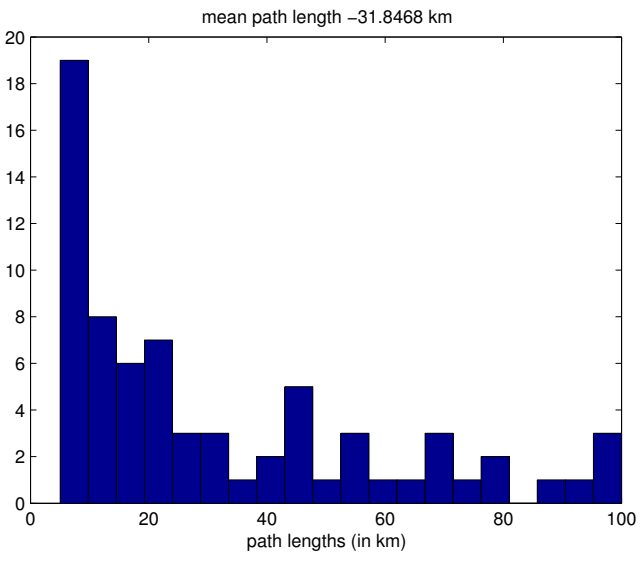

(a) Optimotaxis histogram (mean $\sim 32 \mathrm{~km}$ )

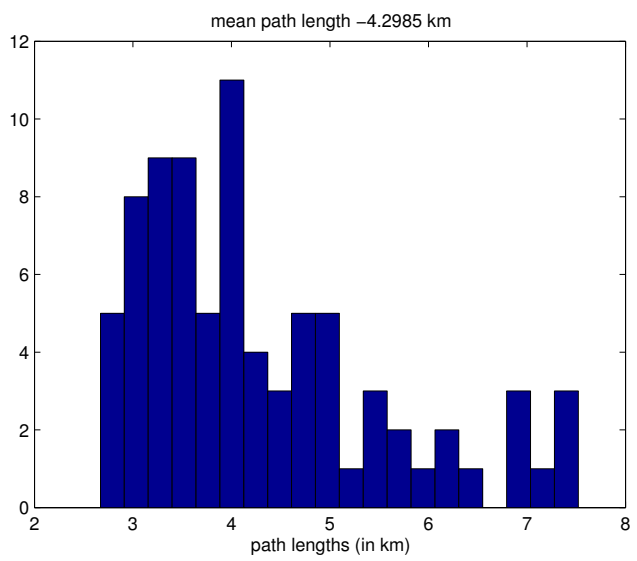

(b) RSS-Optimotaxis histogram (mean $\sim 4.3 \mathrm{~km}$ )

Fig. 12: Histogram of path lengths for optimotaxis and RSSadapted optimotaxis

\begin{tabular}{|c|c|}
\hline Parameter symbol & value \\
\hline \hline$\lambda$ & $0.125 \mathrm{~m}$ \\
\hline$R$ & $80 \lambda=10 \mathrm{~m}$ \\
\hline$T$ & 20 \\
\hline$r_{\text {initial }}$ & $1500 \mathrm{~m}$ \\
\hline$d_{\text {terminate }}$ & $150 \mathrm{~m}$ \\
\hline$d_{\text {walk }}$ & $20 \mathrm{~m}$ \\
\hline$d_{V}$ & $150 \mathrm{~m}$ \\
\hline$d_{H}$ & $25 \mathrm{~m}$ \\
\hline$s_{w}$ & $150 \mathrm{~m}$ \\
\hline$I_{o}$ & $150 \mathrm{~m}$ \\
\hline$d_{\text {sprint }}$ & $300 \mathrm{~m}$ \\
\hline
\end{tabular}

TABLE II: Simulation parameters

UAV to traverse a total distance which is an order of magnitude longer than the shortest path from its initial location to the beacon. Our RSS-adapted optimotaxis algorithm avoids the many false turns taken by general-purpose optimotaxis by expending significant effort in determining a new direction to go in when progress in the current direction becomes unsatisfactory, exploiting the dependence of spatial frequency on approach angle. The distance travelled when using the 


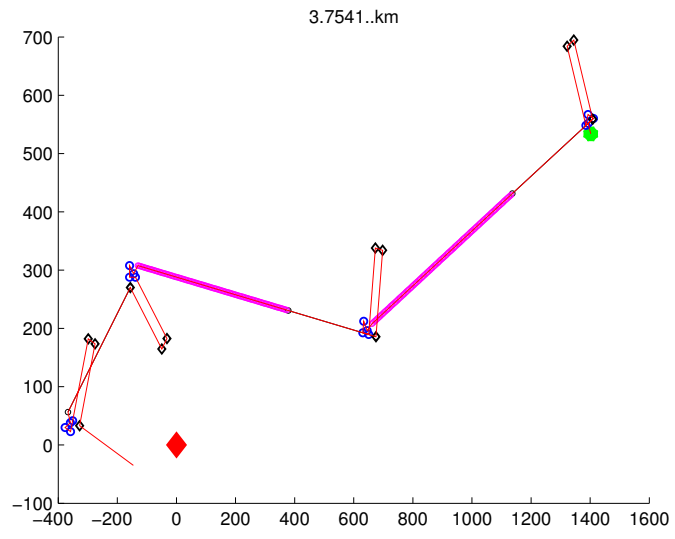

(a) Total tracking path length $-3.8 \mathrm{~km}$

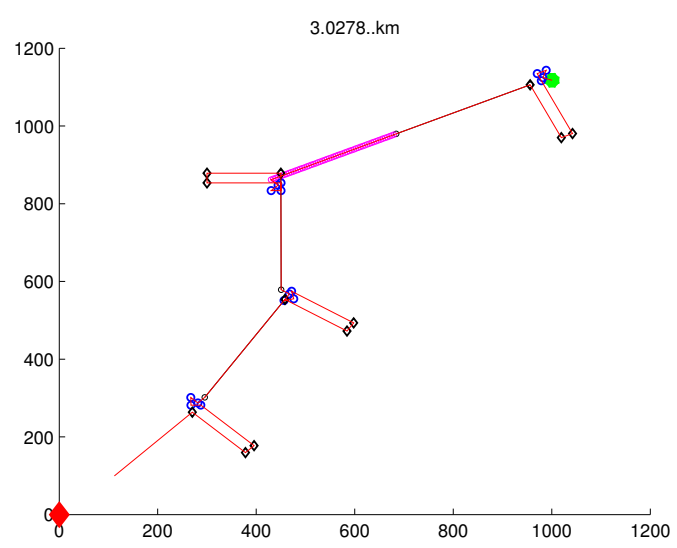

(b) Total tracking path length $-3.0 \mathrm{~km}$

Fig. 13: Two sample tracking paths of RSS-Adapted optimotaxis, with starting distance $r_{\text {initial }}=1.5 \mathrm{~km}$ (green circle is the starting point and source is at the red dot (origin))

proposed algorithm is only a few times larger than the shortest possible distance. Important topics for future research include obtaining more detailed analytical insight into optimization of the steps of the algorithm (e.g., is it possible to obtain significant improvements over the piecewise linear trajectories considered here), exploring sensitivity to noise in the context of detailed link budgets and beaconing schemes, and exploring the effect of changes in propagation geometry (e.g., blockage of the LoS path).

What we have investigated here is perhaps the most difficult of the scenarios of interest. It is of interest to explore how, and to what extent, performance can be improved by using additional information; for example, even coarse angle-ofarrival information may enable us to eliminate Step 2. Another important topic is to explore design and performance for different scattering environments; for example, if both the UAV and the source lie in urban clutter, we may be able to average out fades far more effectively.

\section{REFERENCES}

[1] A. R. Mesquita, J. P. Hespanha, and K. Åström, "Optimotaxis: A stochastic multi-agent optimization procedure with point measurements," Hybrid Systems: Computation and Control, pp. 358-371, 2008.

[2] P. Bahl and V. Padmanabhan, "RADAR: An in-building RF-based user location and tracking system," in INFOCOM 2000. Nineteenth Annual Joint Conference of the IEEE Computer and Communications Societies. Proceedings. IEEE, vol. 2, pp. 775-784, Ieee, 2000.

[3] K. Whitehouse, C. Karlof, and D. Culler, "A practical evaluation of radio signal strength for ranging-based localization," ACM SIGMOBILE Mobile Computing and Communications Review, vol. 11, no. 1, pp. 4152, 2007.

[4] N. Patwari, J. Ash, S. Kyperountas, A. Hero III, R. Moses, and N. Correal, "Locating the nodes: cooperative localization in wireless sensor networks," Signal Processing Magazine, IEEE, vol. 22, no. 4, pp. 54-69, 2005.

[5] J. Wilson and N. Patwari, "Radio tomographic imaging with wireless networks," Mobile Computing, IEEE Transactions on, vol. 9, no. 5, pp. 621-632, 2010.

[6] K. Woyach, D. Puccinelli, and M. Haenggi, "Sensorless sensing in wireless networks: Implementation and measurements," in Modeling and Optimization in Mobile, Ad Hoc and Wireless Networks, 4th International Symposium on, pp. 1-8, IEEE, 2006.

[7] Y. Mostofi, M. Malmirchegini, and A. Ghaffarkhah, "Estimation of communication signal strength in robotic networks," in Robotics and Automation (ICRA), 2010 IEEE International Conference on, pp. 19461951, IEEE, 2010.

[8] J. Fink and V. Kumar, "Online methods for radio signal mapping with mobile robots," in Robotics and Automation (ICRA), 2010 IEEE International Conference on, pp. 1940-1945, IEEE, 2010.

[9] H. Xia, H. Bertoni, L. Maciel, A. Lindsay-Stewart, and R. Rowe, "Radio propagation characteristics for line-of-sight microcellular and personal communications," Antennas and Propagation, IEEE Transactions on, vol. 41, no. 10, pp. 1439-1447, 1993.

[10] T. Stoyanova, F. Kerasiotis, A. Prayati, and G. Papadopoulos, "Evaluation of impact factors on rss accuracy for localization and tracking applications," in Proceedings of the 5th ACM international workshop on Mobility management and wireless access, pp. 9-16, ACM, 2007.

[11] P. Yu, J. Twigg, and B. Sadler, "Radio signal strength tracking and control for robotic networks," in Proceedings of SPIE, vol. 8031, p. 803116,2011

[12] P. Yu and B. Sadler, "Received signal strength gradient estimation for mobile networks," in MILITARY COMMUNICATIONS CONFERENCE 2010 - MILCOM 2010, pp. 519-523, 2010.

[13] Y. Sun, J. Xiao, X. Li, and F. Cabrera-Mora, "Adaptive source localization by a mobile robot using signal power gradient in sensor networks," in Global Telecommunications Conference, 2008. IEEE GLOBECOM 2008. IEEE, pp. 1-5, IEEE, 2008.

[14] D. Han, D. Andersen, M. Kaminsky, K. Papagiannaki, and S. Seshan, "Access point localization using local signal strength gradient," Passive and Active Network Measurement, pp. 99-108, 2009.

[15] R. Clarke, "A statistical theory of mobile-radio reception," Bell Syst. Tech. J, vol. 47, no. 6, pp. 957-1000, 1968.

[16] S. Saunders and A. Aragón-Zavala, Antennas and propagation for wireless communication systems. Wiley, 2007. 